Obstetrics and Gynecology 14, 488 (1959). - 13. Lewish JR., J., Amer. J. Obst. Gynec. 84, 1408 (1962). - 14. Wintersberger, E. and H. TUPPY, Mh. Chem. 91, 406 (1960). - 15. Tuppy, H. and E. Wintersberger, Mh. Chem. 91, 1001 (1960). - 16. Porath, J. and P. Flodin, Nature 183, 1657 (1959). - 17. Flodin, P. and J. Killender, Biochem. Biophys. Acta 63, 403 (1962). - 18.
Tiselius, A., S. HJerten and O. Levin, Arch. Biochem. Biophys. 65, 132 (1956). - 19. StoklasKa, E. and E. Wintersberger, Arch. exp. Path. Pharmak. 236, 358 (1959). - 20. PAGE, E. W., M. A. Tirus, G. Mohun and M. B. Glendening, Amer. J. Obst. Gynec. 82, 1090 (1961). - 21. SEMM, K. and E. WAIDL, Z. Geburtshilfe Gynaek. 158, 165 (1962).
Professor Dr. F. K. Beller, 550 First Ave.

New York, N. Y. 10016, USA

\title{
Polarographische Untersuchungen der elektrophoretisch aufgetrennten Albuminfraktion
}

\author{
Von S. ZimMERMANN \\ Aus der Medizinischen Klinik der Karl-Marx-Universität Leipzig (Direktor: Prof. Dr. med. R. Emmrich)
}

(Der Schriftleitung zugegangen am 19. November 1964)

\begin{abstract}
Eine von HoMolka angegebene kombinierte elektrophoretisch-polarogtaphische Untersuchung der Serumproteine wurde modifiziert. Es wurden 14 gesunde Versuchspersonen und 36 Patienten mit verschiedenen Krankheitsbildern untersucht und das Ergebnis mit dem der Papierelektrophorese verglichen. Es ergab sich keine höhere Empfindlichkeit für das Vorliegen entzündlicher Prozesse gegenüber der üblichen Papierelektrophorese. Es ließen sich qualitativ bedingte Denaturierungsveränderungen in der elektrophoretisch aufgetrennten Albuminfraktion nachweisen. Die Ursachen werden diskutiert, wobei auf die mögliche Bedeutung der Mukoproteine hingewiesen wird.
\end{abstract}

A combined electrophoretic-polarographic study of serum proteins, described by Homolka, was modified. 14 healthy persons and 36 patients with various illnesses were studied and the results compared with those obtained by paper electrophoresis. It was no more sensitive in the detection of inflammatory conditions than the normal paper electrophoresis. Qualitative denaturation changes were found in the electrophoretically separated albumin fraction. The reasons for this are discussed and it is concluded that the mucoproteins may be responsible.

Die Bestimmung der Aktivität entzündlicher Prozesse ist ein dringendes Problem, das sowohl für die Frühdiagnose als auch für die Therapie zunehmend an Bedeutung gewinnt. Gesucht werden hochempfindliche Methoden, die klinisch und labormäßig weitgehend stumm verlaufende Prozesse anzeigen können. Solche Indikatoren spielen auf dem Gebiete der theumatischen Erkrankungen eine Rolle, doch sind sie auf anderen Gebieten der Medizin von nicht zu unterschätzender Bedeutung. - Unter diesem Gesichtspunkt wurde eine von Homolka (1) angegebene kombinierte elektrophoretisch-polarographische Quantitäts- und Qualitätsbestimmung der Serumeiweißfraktionen, die empfindlicher als die übliche Papierelektrophorese sein sollte, geprüft.

\section{Methodik \\ Prinzip}

Die Serumeiweiße werden in der Papierelektrophorese aufgetrennt und die einzelnen Fraktionen nach ihrer Elution auf ihre Denaturierbarkeit polarographisch untersucht, wobei die aufschlußreichsten Veränderungen sich in der Albumin-Fraktion finden, die deshalb auch allein weiter verfolgt werden soll. Mit dieser Methode werden sowohl qualitative als auch quantitative Veränderungen der einzelnen Eiweißfraktionen summarisch erfaßt. Es ist bekannt (2), und es läßt sich im Versuch leicht zeigen, daß polarographische Aktivität der einzelnen Serumeiweißfraktionen nicht nur von ihrer Qùàlität, sondern auch von ihrer Quantität abhängig ist, wobei besonders zu beachten ist, da $\beta$ die Freisetzung nach Alkaligabe nicbt der Eiweißmenge linear proportional erfolgt. In dem hier interessierenden Bereich $z$ wischen 20 und $40 \mu \mathrm{g}$ Albumin steigt die Freisetzung mit zunehmender Menge deutlich stärker an. Bei $50 \mu \mathrm{g}$ wird ein Maximum an Freisetzung erreicht. Größere Eiweißmengen führen wieder zu einer relativen Verminderung der Freisetzung.

Wenn in der elektrophoretisch aufgetrennten Albuminfraktion eine erniedrigte Freisetzung polarographisch aktiver Gruppen nach Alkalidenaturierung gefunden wird, dann kann das sowohl durch eine Verminderung der Eiweißmenge als auch durch qualitative Veränderungen der im Albuminbereich gelegenen polarographisch wirksamen Gruppen bedingt sein. Eine Differenzierung ist nicht möglich. Es interessierte zunächst, ob und in welchem Umfang zusätzliche qualitative Änderungen in der elektrophoretisch aufgetrennten Albuminfraktion bei entzündlichen Prozessen sich nachweisen ließen. Aus diesem Grunde wurden die nach papierelektrophoretischer Auftrennung exhaltenen Proteinfraktionen vor dem Polarographieren jeweils auf einen 
gleichen Eiweißgehalt von $40 \mu \mathrm{g}$ eingestellt, um quantitätbedingte Unterschiede der Denaturierung weitgehend auszuschließen. - Als Maß der Freisetzung wurde der Quotient aus der Höhe der polarographischen Welle nach Denaturierung dividiert durch die Höhe der polarographischen Welle vor Alkalidenaturierung gewählt.

Mit dieser Methodik wurden 50 Seren, 14 gesunde Versuchspersonen und 36 Patienten mit verschiedenen Krankheitsbildern, untersucht und das Ergebnis mit dem Resultat der üblichen Papierelektrophorese verglichen.

Zur Methodik der kombinierten elektrophoretisch-polarographischen Untersuchung ist zu betonen, worauf auch НомоцкA (1) schon hinweist, daß sie einen großen Arbeitsaufwand erfordert und daß sehr exaktes Arbeiten notwendig ist. Aber auch bei peinlicher Einhaltung aller Vorschriften sind noch Fehlermöglichkeiten gegeben, z. B. schon dadurch, daß die elektrophoretische Auftrennung auf dem zu eluierenden Streifen dem gefärbten Teststreifen nicht immer-streng parallel geht.

\section{Technik}

Papierelektrophorese nach Grassmann und Hannig (3) $0,03 \mathrm{~m} /$ Serum, Laufzeit $16 \mathrm{Stdn}$., Spannung 110 Volt; Michaelispuffer $\mathrm{pH}=8,6$, Ionenstärke 0,1; Papier: Niederschlag „FN 3“.

Gesamteiweißbestimmung mittels Biuret-Methode (4)

\section{Polarographie}

Kathodische Polarisation von 1,3-1,7 Volt; Empfindlichkeit 1/50; tropfende $\mathrm{Hg}$-Kathode, feste Silberanode; Tropfzeit 3 Sek. Aufnahme des 1. Polarogramms nach Elution der Proteinfraktion und Einstellung auf $40 \mu \mathrm{g}$ Eiweißgehalt sofort nach Alkalizugabe (1-n KOH) mittels CoIII-Lösung nach BRDičKA. Aufnahme des 2. Polarogramms 45 Min. nach Alkalizugabe.

\section{Isolierung der Mukoproteine}

Serumfiltrat nach Perchlorsäurefällung (wie nach WINZLER) wird im Unterdruckverfahren auf Serumdichte eingeengt und gegen physiologische Kochsalzlösung dialysiert.

Ablenkungselektrophorese nach GRASSMANN und HANNIG (5)

Auftrennung von etwa $3,0 \mathrm{ml}$ Serum; Laufzeit $20 \mathrm{Stdn}$; Spannung 10 Volt/cm; Papier: Schleicher und Schüll 2040a.

\section{Ergebnisse und Diskussion}

Der Index der Freisetzung schwankte zwischen 5,6 und 1,3. Die Verteilung in den einzelnen Patientengruppen zeigt Tabelle 1. Als Grenzwert wurde ein Quotient von 1,8 angenommen. Werte unter 1,8 galten als pathologisch, Werte über 1,8 als normal. Der Wert 1,8 entspricht der Grenze des statistischen Vertrauensbeweises (arithmetisches Mittel $-2 \sigma$ ). Jeder Einzelwert der Tabelle 1 entspricht dem arithmetischen Mittel von Doppel- oder Dreifachbestimmungen.

Die unterschiedliche Höhe der Freisetzung nach Alkalidenaturierung zeigt eindeutig, daß qualitative Veränderungen in der Albuminfraktion vorhanden sein müssen, die in der ursprünglichen Versuchsanordnung von HoмOLKA neben den quantitativbedingten Veränderun-
Tab. 1

Polarographischer Freisetzungsindex bei Gesunden und Patienten mit und ohne Dysprotcinämie

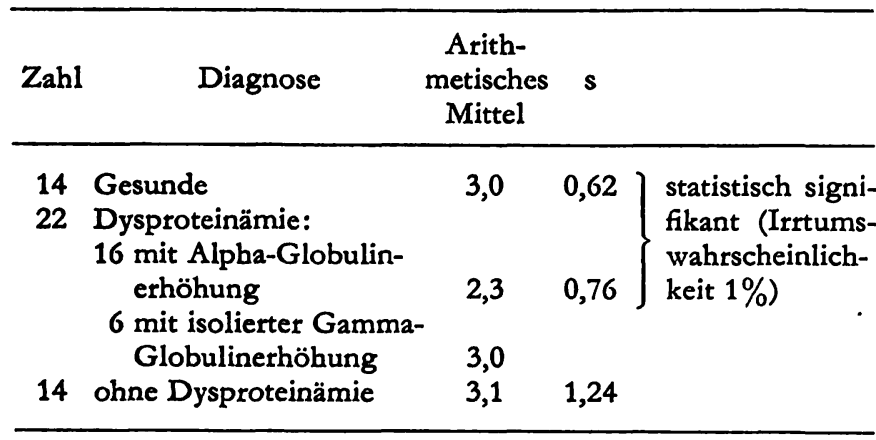

gen wirksam werden. Worauf die unterschiedliche Denaturierbarkeit zurückzuführen ist, ist noch nicht geklärt. Von Homolka wurden qualitative Veränderungen des Albumins angenommen. So wurden unterschiedliche Mengenänderungen freier basischer Gruppen, Veränderungen der Metallbindungsfähigkeit und abweichende serologische Eigenschaften als Ursache vermutet. Auch muß an eine Beeinflussung des Albumins durch in das Blut ausgeschwemmte proteolytische Enzyme gedacht werden. Andererseits sind die bis jetzt eindeutig bekannten Veränderungen des Albumins rein quantitativer Natur. Eine Mikroheterogenität des Albumins wird zwar diskutiert (WUHRMANN und MärKI (6)), ist aber keineswegs exakt bewiesen. Sie kann vorgetäuscht werden, da Albumin der Träger zahlreicher körpereigener und körperfremder Substanzen ist. So kann die veränderte Denaturierbarkeit durch zusätzliche mit dem Albumin wandernde Substanzen bedingt werden. In der elektrophoretisch aufgetrennten Albuminfraktion liegen außer dem Albumin noch zahlreiche andere, gleich schnell wandernde Substanzen vor, zum Beispiel findet sich ein Teil der polarographisch als aktiv bekannten Mukoproteine in der Albuminfraktion. $\mathrm{Da} \beta$ die Mukoproteine den Freisetzungsindex deutlich beeinflussen, zeigt die Tabelle 2 .

Tab. 2

Beeinflussung des polarographischen Index durch Zusatz von Mukoproteinen

\begin{tabular}{ccc}
\hline$\mu \mathrm{g}$ Albumin $+\mu \mathrm{g}$ Mukoproteine & $\begin{array}{c}\text { polarographischer } \\
\text { Index }\end{array}$ \\
\hline 40 & 40 & 1,5 \\
40 & 20 & 1,6 \\
40 & 10 & 1,8 \\
10 & 40 & 1,0 \\
20 & 40 & 1,4 \\
\hline
\end{tabular}

Um zu prüfen, ob die Mukoproteine in unserer Versuchsanordnung als Ursache für die Erniedrigung des Quotienten in Frage kommen könnten, wurde die Albuminfraktion mit der kontinuierlichen Ablenkungselektrophorese nach GrassmanN und HaNNIG in weitere Unterfraktionen aufgeteilt. 
Die Tabelle 3 zeigt, daß Mukoproteine in den langsamwandernden Anteilen der Albuminfraktion vorkommen, und daß der Freisetzungsindex in diesen Unterfraktionen geringer ist als in den von Mukoproteinen freien, schnellwandernden Albuminunterfraktionen. Die Ergebnisse weisen daraufhin, daß die Mukoproteine als Ursache der Erniedrigung des polarographischen Index in der Albuminfraktion in Frage kommen können, wobei natürlich andere Ursachen dadurch keineswegs ausgeschlossen worden sind.

\section{Tab. 3}

Der polarographische Index in den Albuminunterfraktionen. Der Index entspricht jeweils dem arithmetischen Mittel aus 8 Einzelbestimmungen

\begin{tabular}{lc}
\hline $\begin{array}{c}\text { schnellwandernde } \\
\text { Fraktion } \\
\text { (frei von Mukoproteinen) }\end{array}$ & $\begin{array}{c}\text { langsamwandernde } \\
\text { Fraktion } \\
\text { (enthält Mukoproteine) }\end{array}$ \\
\hline $\begin{array}{l}\text { Polarographischer } \\
\text { Index }\end{array}$ & 2,5 \\
\hline
\end{tabular}

Für die klinische Praxis interessierte besonders, ob die kombinierte polarographisch-elektrophoretische Untersuchung ein empfindlicherer Indikator entzündlicher Prozesse als die üblichen Untersuchungen ist. $\mathrm{Zu}$ dieșem Zwecke wurden die Ergebnisse mit den bei der Papierelektrophorese erhaltenen Befunden verglichen: Tabelle 4.

Die Tabelle 4 zeigt, daß bei 37 von 50 untersuchten Seren die Ergebnisse einander entsprachen. 31 Seren zeigten weder im Elektropherogramm noch im polarographischen Index ein von der Norm abweichendes Verhalten. In 6 Fällen fand sich bei einer Erhöhung der Alpha-Globuline ein pathologisch erniedrigter polarographischer Index. 11 Seren zeigten eine Erhöhung der Alpha-2-Globuline, während der polarographische Index noch keine eindeutige Abweichung erkennen ließ. Bei
Tab. 4

Vergleich zwischen Dysproteinämie und polarographischem Index

\begin{tabular}{cccc}
\hline $\begin{array}{c}\text { Anzahl der } \\
\text { untersuchten } \\
\text { Seren }\end{array}$ & $\begin{array}{c}\text { Erhöhung } \\
\text { der Alpha- } \\
\text { Globuline }\end{array}$ & $\begin{array}{c}\text { Isolierte } \\
\text { Erhöhung der } \\
\text { Gamma-Globuline }\end{array}$ & $\begin{array}{c}\text { Polarogra- } \\
\text { phischer Index } \\
\text { unter 1,8 }\end{array}$ \\
\hline 6 & + & & + \\
11 & + & - & + \\
2 & - & + & + \\
3 & - & + & + \\
1 & - & - & - \\
31 & & - & \\
\hline
\end{tabular}

2 Seren war das Ergebnis umgekehrt; die positive polarographische Reaktion fand sich einmal bei einem klinisch gesunden Blutspender, der ein normales Elektropherogramm aufwies, im 2. Fall handelte es sich um eine ältere Frau mit einer Hypoproteinämie ohne wesentliche Dysproteinämie.

Wenn auch im Durchschnitt der polarographische Index in der Gruppe der Patienten mit Dysproteinämie niedriger ausfällt und der Unterschied zur Gruppe der gesunden Versuchspersonen statistisch noch signifikant ist, so zeigt doch der Vergleich $z$ wischen Papierelektrophorese und kombiniertem polarographisch-elektrophoretischem Verfahren keine höhere Empfindlichkeit der kombinierten Methode an. Vielmehr waren eindeutig entzündliche Veränderungen in der Papierelektrophorese, die dem klinischen Bild parallel gingen, im polarographischen Index stumm.

Die Einführung in die Routinediagnostik als hochempfindlicher Indikator für sonst weitgehend subklinisch verlaufende Prozesse kann daher nicht empfoblen werden. Es kommt hinzu, daß die Methode sehr arbeitsaufwendig ist und auch bei Einhaltung aller Versuchsbedingungen Fehlermöglichkeiten beinhaltet, die auch bei Zwei- und Dreifachbestimmungen nicht ausgeschlossen werden können.

\section{Literatur}

1. Homorka, J., Die elektrophoretisch-polarographische Quantitäts- und Qualitätsbestimmung der Serumeiweißfraktionen, in: BüChner, M., Moderne Chemische Methoden in der Klinik, Georg Thieme Verlag, Leipzig (1961). - 2. Tropp, C., L. JüHLING und F. Geiger, Hoppe-Seyler's Z. physiol. Chem. 262, 225 (1939). 3. GrassmanN, W. und K. Hannig, Naturwissenschaften 37,469
(1950). - 4. Weichelabaum, T. E., Amer. J. clin. Pathol. 10, 40 (1946). - 5. Grassmann, W. und K. Hannig, Hoppe-Seyler's Z. physiol. Chem. 292, 32 (1953). - 6. WürrmanN, F. und H. H. Märkr, Dysproteinämien und Paraproteinämien, Vetlag Schwabe \& Co., Basel/Stuttgart (1963).
Dr. med. S. Zimmermann Medizinische Universitätsklinik der Karl-Marx-Universität Leipzig C 1, Johannisallee 32 